\title{
ENDOMETRIOSIS VESICAL: DOS NUEVOS CASOS
}

\author{
J.M. ALAPONT ALACREU, A. ANDREU GARCÍA, E. HERRERO POLO, \\ R. BOTELLA ALMODÓVAR, E. SCHIEFENBUSCH MUNNE, J.L. BROTONS MÁRQUEZ, \\ G. LLAMAZARES CACHÁ
}

Servicio de Urología. Hospital General de Elda. Alicante.

Actas Urol Esp. 28 (10): 789-791, 2004

\section{RESUMEN}

ENDOMETRIOSIS VESICAL: DOS NUEVOS CASOS

La endometriosis en el aparato urinario es una patología infrecuente, siendo la vejiga el órgano afecto en la mayoría de las ocasiones. Se manifiesta clínicamente con urgencia, frecuencia, dolor hipogástrico y en ocasiones con hematuria. La cistoscopia es la prueba diagnóstica más fiable aunque el diagnóstico de confirmación es histológico.

Presentamos 2 casos de endometriosis vesical en 2 mujeres jóvenes, una con antecedentes de manipulación ginecológica, en las que el tratamiento quirúrgico fue satisfactorio. Tras 1 y 3 años de seguimiento las pacientes permanecen asintomáticas.

PALABRAS CLAVE: Endometriosis. Vejiga. Cistitis.

\section{ABSTRACT}

VESICAL ENDOMETRIOSIS: REPORT OF TWO CASES

Endometriosis affecting the urinary tract is very rare and the most common site of involvement is urinary bladder. The clinical features are urgency and frequency, hipogastric pain and hematuria. Cistoscopic examination is the most valuable diagnostic test but definitive diagnosis requires histologic confirmation.

We report 2 cases of endometriosis in 2 young women, one with previously cesarean section, in which surgical treatment was effective. After 1 and 3 years of follow-up respectively the patients remain assymptomatic.

KEY WORDS: Endometriosis. Urinary bladder. Cystitis.

$\mathrm{D}$ efinimos endometriosis como la presencia de tejido endometrial fuera de la cavidad uterina. Es una patología que aparece con mayor frecuencia en mujeres jóvenes, siendo la media de edad en el momento del diagnóstico entre 25-29 años ${ }^{1}$.

La endometriosis se localiza principalmente en los ovarios, ligamentos útero-sacros, peritoneo pélvico, trompas de Falopio, cuello cervical, vagina y fondo de saco de Douglas. En el aparato urinario es infrecuente $(1,2 \%$ de los casos descritos, siendo la vejiga el órgano más afecto $-84 \%-)^{2,3}$.
Presentamos dos casos de endometriosis vesical en 2 mujeres, 1 de ellas sin antecedentes de ningún procedimiento o intervención ginecológica. Realizamos además una revisión de la clínica, diagnóstico y tratamiento de esta excepcional patología urológica.

\section{CASOS CLÍNICOS}

Caso 1: Paciente mujer de 30 años con antecedentes de gastritis que acude a nuestra consulta por referir síndrome miccional irritativo que se acentúa en el periodo menstrual. A la exploración 
física no se evidencia ninguna alteración. Entre las exploraciones complementarias solicitadas destacan: analítica y cultivo de orina: normales; ecografía abdominal (Fig. 1): neoformación intravesical de $4 \mathrm{~cm}$ de diámetro mayor que depende de la pared; cistoscopia (Fig. 2): masa azulada, redondeada, a nivel retrotrigonal, compatible con endometrioma vesical. El estudio ginecológico realizado es compatible con endometriosis ovárica. Se realiza RTU de la lesión vesical, exéresis laparoscópica de los endometriomas ováricos y posterior bloqueo hormonal con análogos de la LH-RH. En el seguimiento, a los 2 años, se detecta un aumento del CA $125(140,1 \mathrm{U} / \mathrm{ml})$ por lo que se realiza ecografía y cistoscopia que evidencian una recidiva de la lesión en la misma localización vesical. Se trata de nuevo con RTU y bloqueo hormonal y tras 1 año de seguimiento la paciente permanece asintomática y sin signos de recidiva endometrial.

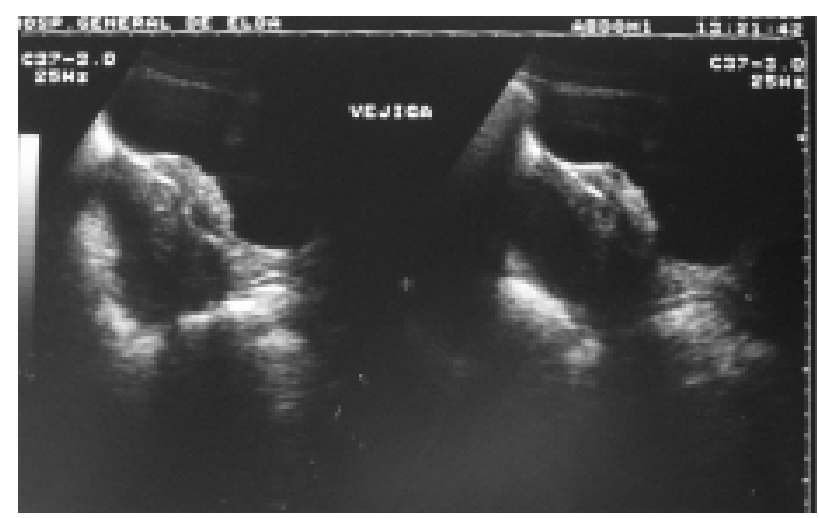

FIGURA 1. Ecografia vesical: Tumoración intraluminal que depende de la pared.

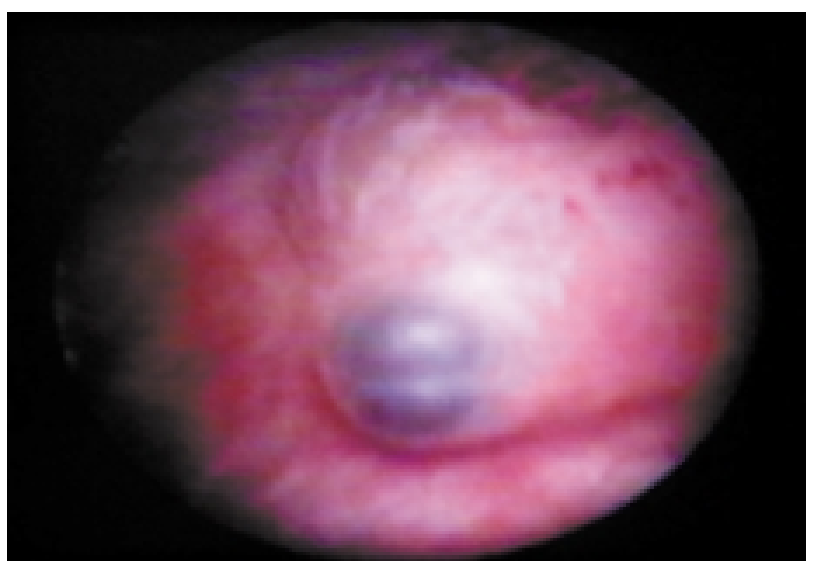

FIGURA 2. Cistoscopia: Endometrioma que protuye en la mucosa vesical.
Caso 2: Paciente mujer de 41 años con antecedentes de psoriasis, hipotiroidismo, ooforectomía derecha y 2 cesáreas. Tras estudio en ginecología por algias pélvicas crónicas se detecta en TC abdómino-pélvica un nódulo de densidad de partes blandas de $3 \mathrm{~cm}$ de diámetro, localizado entre útero y cúpula vesical y que protuye en la luz de ésta; además imagen quística de $2,2 \mathrm{~cm}$ de diámetro en ovario izquierdo. No refería ninguna clínica urológica. La paciente es sometida a una laparotomía exploradora por parte de ginecología realizándose histerectomía, anexectomía izquierda y salpingectomía derecha. Se nos consulta intraoperatoriamente porque la masa dependía de la pared vesical y tras biopsia intraoperatoria (sugestiva de endometriosis) procedimos a resecar toda la pared vesical afecta. El estudio histológico posterior confirmó la lesión endometrial y a los 3 años de seguimiento la paciente está asintomática y sin objetivarse recidiva endometrial.

\section{DISCUSIÓN}

La endometriosis vesical es una rara enfermedad urológica que suele aparecer en mujeres en edad fértil debido a que el tejido ectópico endometrial crece a expensas de los estrógenos. En muy raras ocasiones aparece tras la menopausia (normalmente por aporte exógeno de estrógenos aunque también se han descrito casos con niveles de estrógenos normales en la menopausia o tras la castración) ${ }^{4}$.

La etiopatogenia no está del todo clara, siendo 3 las teorías más aceptadas en la actualidad:

- Teoría embrionaria: a partir de restos de los conductos de Müller y de Wolf.

- Teoría metaplásica: cambios metaplásicos de las células del peritoneo pélvico y de la mucosa vesical.

- Teoría migratoria o de la implantación: paso retrógrado de tejido endometrial a través de las trompas durante la menstruación.

El 84\% de los casos de endometriosis urológica aparecen en la vejiga, localizándose los endometriomas retrotrigonalmente o en la cúpula vesical en la mayoría de las ocasiones.

Las manifestaciones clinicas aparecen de forma cíclica (máxime en la menstruación) en forma de dolor suprapúbico y sindrome miccional irritativo (frecuencia, urgencia, escozor y tenesmo). El signo 
clásico de hematuria durante la menstruación tan sólo aparece en el 20-30\% de las ocasiones ${ }^{5}$. Debemos sospecharla en pacientes con esta sintomatología, urinocultivo negativo y que además han sido sometidas a una manipulación o intervención ginecológica ( $50 \%$ de las ocasiones). En los casos en los que la endometriosis se localiza alrededor de los meatos ureterales se puede manifestar en forma de obstrucción ureteral.

Las técnicas de imagen (ecografía, TC, RNM) pueden demostrar la presencia de una tumoración en la pared vesical. La RNM permite además valorar la extensión de la lesión en la pared vesical y/o órganos de alrededor.

La sospecha clínico-radiológica obliga a realizar una cistoscopia que muestra unas lesiones de aspecto quístico y tinte azulado (Fig. 2). En algunas ocasiones esta exploración es inespecífica, sin embargo está justificada para descartar la presencia de carcinomas del epitelio vesical.

Se ha descrito un aumento del CA 125 en suero de mujeres con endometriosis.

El diagnóstico de confirmación es histológico (presencia de glándulas y estroma endometrial en la pared vesical) mediante biopsia con pinza fría o con el resector ${ }^{6}$.

El tratamiento puede ser médico, quirúrgico o una combinación de ambos y dependerá de la edad de la paciente, tamaño de la lesión, deseo de descendencia y gravedad de los síntomas.

La extirpación quirúrgica de la lesión suele ser el tratamiento de elección en la mayoría de los casos, combinada en ocasiones con el bloqueo hormonal. Se puede realizar resecando transuretralmente los endometriomas en su totalidad o mediante cistectomía parcial (posible laparoscópicamente en algunos casos) asociada a histerectomía y anexectomía en mujeres que no desean descendencia ${ }^{7}$. Lesiones de pequeño y mediano tamaño pueden ser tratadas con láser ${ }^{8}$.

El tratamiento médico estará indicado en aquellos casos en los que la cirugía esté contraindicada o cuando quede tejido residual tras ésta.
Dado que el tejido endometrial ectópico es hormonodependiente este tratamiento consiste en el bloqueo hormonal. Actualmente los análogos de la LH-RH (bloqueantes de la secreción hormonal a nivel central) son los fármacos de elección ${ }^{9}$. Creemos que deben administrarse siempre tras la RTU debido a que la lesión es transmural y existen muchas posibilidades de que quede una pequeña cantidad de tejido endometrial residual. En el caso 1 que presentamos se produjo recidiva pese al tratamiento hormonal y quizá pudo deberse a que no realizamos una RTU de toda la pared como sí que hicimos en la segunda ocasión.

\section{REFERENCIAS}

1. Olive DL, Haney AF. Endometriosis. En DeCherney AH (Ed). Reproductive failure. Churchill Livingstone. New York 1986; 153-203.

2. Abeshouse BS, Abesgause G. Endometriosis of the urinary tract. J Int Coll Surg 1960;34:43-63.

3. Shook TE, Nyberg LM: Endometriosis of the urinary tract. Urology 1988;31:1-6.

4. Habuchi T, Okagaki T, Miyakawa M. Endometriosis of bladder after menopause. J Urol 1991;145:361-363.

5. Moore TD, Herring AL, Mccannel DA. Some urologic aspects of endometriosis. J Urol 1943;49:171-176.

6. Price DT, Maloney KE, Ibrahim GK Cundiff GW, Leder RA, Anderson EE. Vesical endometriosis: report of two cases and review of the literature. Urology 1996;48:639-643.

7. Romero P, Lobato JJ, Pérez LA, Mira A: Endometriosis de la capa muscular vesical como causa de abdomen agudo. Arch Esp de Urol 1995;48:395-397.

8. Vicente J, Laguna P, Díaz I, Algaba F, Calaf J. Tratamiento de la endometriosis vesical con Nd-Yag láser. Arch Esp de Urol 1991;44:169-171.

9. García J, Fernández JM, Jalón A, Martín JL, Rodríguez O, Rodríguez JJ, San Martín A, Martínez FJ, Sánchez A, Regadera J: Nuevo caso de endometriosis vesical en paciente con antecedentes de cesárea. Arch Esp de Urol 2003;56:952-954.

Dr. J.M. Alapont Alacreu

Servicio de Urología

Hospital General de Elda

Elda-Sax, La Torreta, s/n

03600 Elda (Alicante)

(Trabajo recibido el 7 enero de 2004) 